This item is the archived peer-reviewed author-version of:

\title{
Quantitative tomography of organic photovoltaic blends at the nanoscale
}

\section{Reference:}

Pfannmöller Martin, Heidari Mezerji Hamed, Nanson L., Lozman O.R., Chrapa M., Offerrnans T., Nisato G., Bals Sara.Quantitative tomography of organic photovoltaic blends at the nanoscale

Nano letters / American Chemical Society - ISSN 1530-6984 - 15:10(2015), p. 6634-6642

Full text (Publishers DOI): http://dx.doi.org/doi:10.1021/ACS.NANOLETT.5B02437

To cite this reference: http://hdl.handle.net/10067/1294230151162165141 


\section{Quantitative tomography of organic photovoltaic blends at the nanoscale}

M. Pfannmöller*1, H. Heidari*1, L. Nanson², O. R. Lozman², M. Chrapa ${ }^{3}$, T. Offermans ${ }^{3}$, G. Nisato ${ }^{3}$, S. Bals $^{\# 1}$

* The authors contributed equally to this work.

1 EMAT, Antwerp University, Antwerp, Belgium

2 Merck Chemicals Ltd, Chilworth Technical Centre, Southampton, UK

3 CSEM, Muttenz, Switzerland

\# Corresponding author e-mail address: sara.bals@uantwerpen.be

Keywords: Organic solar cells; Amorphous polymers; Spectral tomography; On-axis tomography; Electron energy-loss spectroscopy; Spectroscopic imaging

\section{Abstract}

The success of semiconducting organic materials has enabled green technologies for electronics, lighting, and photovoltaics. However, when blended together, these materials have also raised novel fundamental questions with respect to electronic, optical, and thermodynamic properties. This is particularly important for organic photovoltaic cells based on the bulk heterojunction. Here, the distribution of nanoscale domains plays a crucial role depending on the specific device structure. Hence, correlation of the aforementioned properties requires 3D nanoscale imaging of materials domains, which are embedded in a multilayer device. Such visualization has so far been elusive due to lack of contrast, insufficient signal or resolution limits. In this article, we introduce spectral scanning transmission electron tomography for reconstruction of entire volume plasmon spectra from rodshaped specimens. We provide 3D structural correlations and compositional mapping at a resolution of better than $7 \mathrm{~nm}$ within advanced organic photovoltaic tandem cells. Novel insights that are obtained from quantitative 3D analyses reveal that efficiency loss upon thermal annealing can be attributed to subtle, fundamental blend properties. These results are invaluable in guiding the design and optimization of future devices in plastic electronics applications and provide an empirical basis for modelling and simulation of organic solar cells. 
During the last few decades, electron tomography has evolved into a standard tool to investigate the three-dimensional (3D) morphology or structure of nanometer-sized objects, even at atomic resolution ${ }^{1,}$ 2. More recently, 3D chemical distributions were visualized by combining the concept of tomography with energy dispersive X-ray spectroscopy ${ }^{3,4}$ or electron energy-loss spectroscopy $(E E L S)^{5-7}$. The latter approach, here referred to as "spectral tomography" (ST), was used to investigate a broad range of materials, such as $\mathrm{CeO}_{2}$ nanoparticles ${ }^{8}$ or silver nanoparticles using a 3D reconstruction of surface plasmon resonances ${ }^{9}$. Furthermore, 3D reconstructions of EEL data acquired in the low-loss regime made it possible to differentiate between the signals from silicon and silicon oxide ${ }^{10}$ or between carbonnanotubes and a nylon host matrix ${ }^{11}$. This latter approach was also applied for thin films consisting of blends of very similar organic semiconductors, which form bulk heterojunction nanoscale networks used in organic photovoltaics (OPV) $)^{12,13}$. However, the bulk heterojunction morphology depends heavily on the existing interfaces that are present. Therefore it is of crucial importance to image the organic materials within the specific device stack in which the performances are evaluated ${ }^{14,15}$ and which has so far been neglected by the considerable majority of studies. Even with quantitative methods such as scanning transmission X-ray microscopy significant morphological features of the order of $10 \mathrm{~nm}$ and below cannot be visualized ${ }^{16}$. In this article we demonstrate that these limitations can be overcome by ST of plasmon excitations. A key requirement for reliable 3D reconstructions is a monotonic signal increase upon tilting. When tilting lamellar samples prepared by focused ion beam (FIB) milling, the projected thickness rapidly increases, so that this requirement may not be fulfilled anymore for such FIB samples. Moreover, tilting over a range of $\pm 90^{\circ}$ is impossible for transmission electron microscopy (TEM) lamellas, leading to so-called missing-wedge artefacts, which hamper a reliable quantification of the final reconstruction ${ }^{17,18}$. Here, we have used FIB milling to prepare rod-shaped specimens such that the thickness of the TEM sample remains constant throughout the complete tilt series to retrieve a 3D reconstruction of the entire OPV device without missing wedge artefacts ${ }^{19}$. An even more fundamental 
problem is related to the lack of contrast between the active layers in the OPV device, which typically contain a polymer:fullerene blend. Due to the close similarity of the materials, it is far from straightforward to acquire projection images in which sufficient contrast is realised ${ }^{20}$. By 3D reconstruction of the entire EEL spectra that show specific volume plasmon signals we were able to generate material contrast at a spatial resolution of around $7 \mathrm{~nm}$. We observe that a device efficiency drop after thermal heating originates from subtle morphological changes.

Using the full information available from reconstructions of plasmon spectra we are able to extend qualitative visualization to 3D quantitative chemical mapping, unravel preferential mixing ratios within the organic blends and relate structural changes to the materials properties. We envisage that this approach will lead to a better understanding of the different parameters that determine the efficiency and lifetime of these devices including all layers of an optimized stack design. It will be particularly important for the recent trend towards high-efficiency devices incorporating mainly amorphous polymers ${ }^{21}$, where compositional mapping at the sub-10 $\mathrm{nm}$ scale has been elusive so far-even in twodimensional (2D) imaging.

The approach that we propose here is used to investigate OPV tandem cell devices having a power conversion efficiency of $6-7 \%$. The tandem cells contain two single cells with different polymer:fullerene blends. One cell includes a novel high band-gap polymer (HBG1), a high performing thiophene-rich conjugated donor-acceptor polymer. A loss in performance for more than $10 \%$ was found in the tandem cells when comparing heated and non-heated samples. In order to obtain a better understanding concerning the origin of this loss, we investigated the nanoscale morphology of the entire device.

The device architecture is schematically presented in Figure 1a. In the configuration of the devices, calcium (Ca), and the polymer mixture PEDOT:PSS act as interfacial layers to extract electrons and holes, respectively, at the interfaces. The central layer serves as recombination layer and consists of a small layer of PEDOT:PSS on top of colloidal ZnO. The latter transports electrons from the bottom cell and the 
former holes from the top cell for recombination (cf. Figure 1a). The two solar cells are composed of the polymer $\mathrm{PDPP}^{22}$ in conjunction with the fullerene derivative $\mathrm{PC}_{70} \mathrm{BM}^{23}$ in the top layer, and the polymer $\mathrm{HBG1}$ in conjunction with the fullerene derivative $\mathrm{PC}_{60} \mathrm{BM}$ in the bottom layer. Whilst we cannot disclose the structure of the polymer HBG1, we can make the materials available at commercial rates to anyone that wishes to explore further. Fullerenes act as electron acceptor materials whereas holes are transported in the polymeric donor materials. Rod-shaped samples were prepared using a FIB protocol accounting for the device architecture-the combination of soft and hard layers may lead to preferential milling and specific charging behaviour. The protocol includes the use of low currents for thinning of the rod and patterns to produce a cone shape, which is required not to charge the sample under the electron beam (cf., Supporting information).

An overview (Figure 1b) of the FIB-prepared rod-shaped specimen of the non-heated device was acquired by high-angle annular dark field scanning transmission electron microscopy (HAADF-STEM).

The HAADF-STEM intensity depends on the atomic number $Z$ of the constituent elements within the layer, resulting in a higher intensity for layers that contain elements with higher $Z$. This enables us to investigate the interfaces between the different layers in the device.
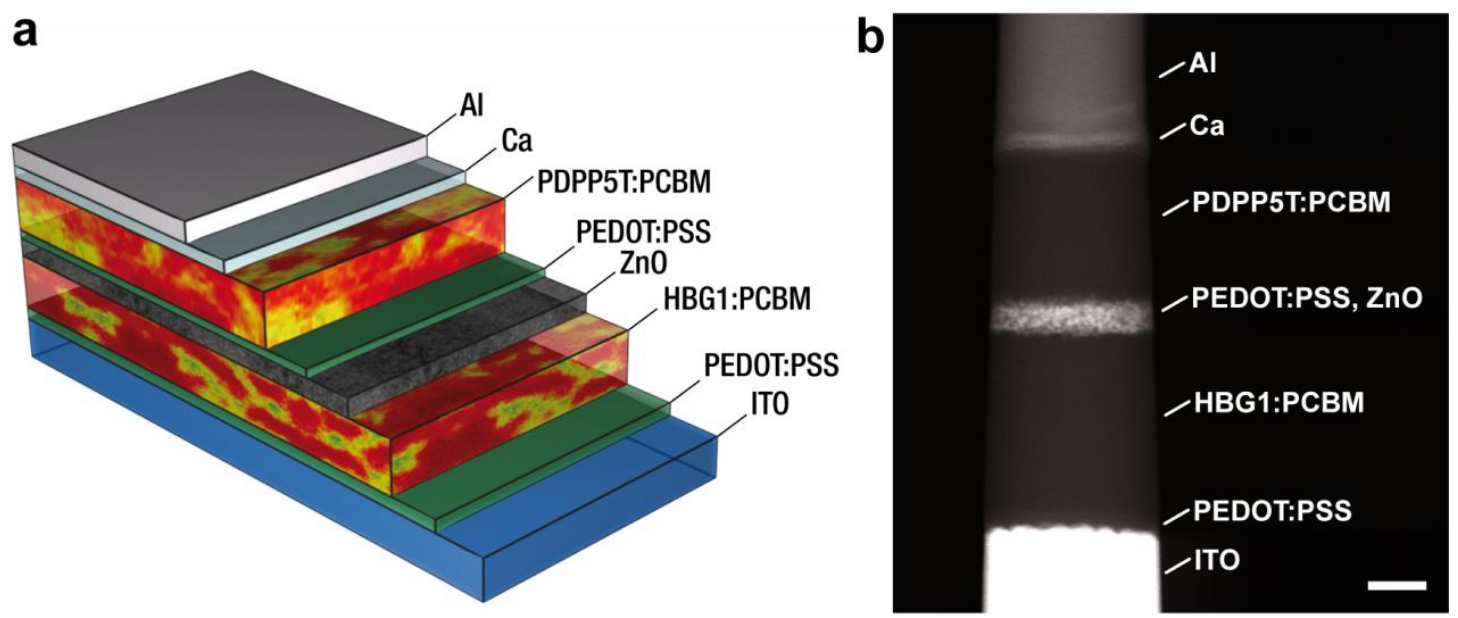

Figure 1: Structure of investigated tandem cell stack. a: Schematics of the different layers of the investigated samples. It is expected that the full device including the bottom photoactive layer ( $\left.\mathrm{HBG1}: \mathrm{PC}_{60} \mathrm{BM}\right)$ and top active layer (PDPP5T:PC $\left.70 \mathrm{BM}\right)$, the recombination layer (ZnO, PEDOT:PSS), anode (PEDOT:PSS, ITO), and cathode (Ca, Al) favours a particular nanomorphology of the carbonaceous layers. b: Conventional dark-field STEM image of rod cut perpendicular to the device layers using a FIB. The 
layer materials are specified in this image. No material contrast within the photoactive layers is observed. Scale bar in $\mathbf{b}$ represents $50 \mathrm{~nm}$.

Unfortunately, HAADF-STEM is unable to provide reliable contrast between the organic domains ${ }^{20}$. We therefore applied low-loss EEL spectral imaging (see supporting information for technical details). Using scanning TEM (STEM), spatially resolved spectra of the low-energy-loss region are sequentially recorded while scanning across the specimen. Since EEL spectra with high energy resolution can be extracted for each scan position, images with information for specific energy windows can be generated. Figure $2 a$ shows a $2 \mathrm{D}$ view of the rod from the non-heated device with contrast information averaged between 26-30 eV. Here, polymer-enriched phases appear darker due to the lower energy position of volume plasmons compared to fullerenes. Figure $2 \mathrm{~b}$ depicts average spectra taken from dark (polymer-rich) and bright (fullerene-rich) regions in Figure 2a for both cells in the tandem structure. The main feature in the spectra is the bulk plasmon $(\pi+\sigma)$ with a maximum at energies higher than $20 \mathrm{eV}$. It was shown by Schindler et al. that fitting bulk plasmon signals, extracted from energy-filtered TEM, with a Gaussian provides morphological maps ${ }^{24}$. Here, for qualitative maps of domain compositions, we fitted all spectra from a spectral data set with a Lorentzian function. The dotted lines in Figure $2 b$ show Lorentzian fits to the $(\pi+\sigma)$ volume plasmons. Peak energies are approximately $23.4,23.7,22.5,24.6$, and $24.5 \mathrm{eV}$ for areas rich in $\mathrm{HBG1}$, PDPP5T, PEDOT:PSS, $\mathrm{PC}_{60} \mathrm{BM}$, and $\mathrm{PC}_{70} \mathrm{BM}$, respectively. The exact fits are possible by applying a deconvolution prior to fitting, which removes artefacts due to multiple scattering of the electrons at thicker portions of the sample ${ }^{25}$ (see Supporting information). Low-loss spectra from the inorganic layers ( $\mathrm{Al}, \mathrm{Ca}, \mathrm{ZnO}$ and ITO) are presented in Figure 2c. These spectra yield unique features and can be readily separated from polymer and fullerene spectra. A Lorentzian fit to the $\mathrm{ZnO}$ spectrum reveals that the spectrum is not pure in this projection data but is a superposition of different signals.

Figure $2 \mathrm{~d}$ and $2 \mathrm{e}$ represent-in false colour-2D visualizations of the non-heated and heated device morphologies, respectively, which are obtained by mapping the Lorentzian centre positions. Differences in centre position relate to differences in plasmon frequencies of polymers and fullerenes. It has been 
shown in various works and for different polymers that peak positions are found at ca. 21.5-22 eV for polymers and around $25-26 \mathrm{eV}$ for fullerenes ${ }^{13,26-28}$. Therefore we can assign small values of centre positions within the photoactive layers to polymer-rich areas (green) and larger values to fullerene-rich areas (red). As a polymer mixture of two ionomers, PEDOT:PSS also shows low peak energies. Since PEDOT:PSS forms a pure layer between the ITO and the bottom photoactive layer, it is visualized in projection as green to even light-blue, corresponding to energies of ca. $22.5-21.5 \mathrm{eV}$ in the presented maps. These maps provide qualitative information about demixing and information about the vertical distribution of the polymer vs. fullerene compound within the photoactive layers of the devices. The increase in the amount of green parts in Figure $2 \mathrm{e}$ suggests that the additional heating step induces a certain amount of demixing. However, since only a mild demixing is observed, it does not explain the drop in performance. A careful comparison of the morphologies for the non-heated and heated device also indicates a reduction of the fullerene content near the $\mathrm{ZnO}$ upon heating. Decrease in efficiency upon heating was found to be mainly due to a drop in open-circuit voltage. Consequently, movement of fullerenes away from the recombination layer would better explain this behaviour due to space charge accumulations. However, it is still possible that small domains of fullerene protrude towards $\mathrm{ZnO}$ for transport of electrons, but such conclusions cannot be drawn from a single 2D projection. 

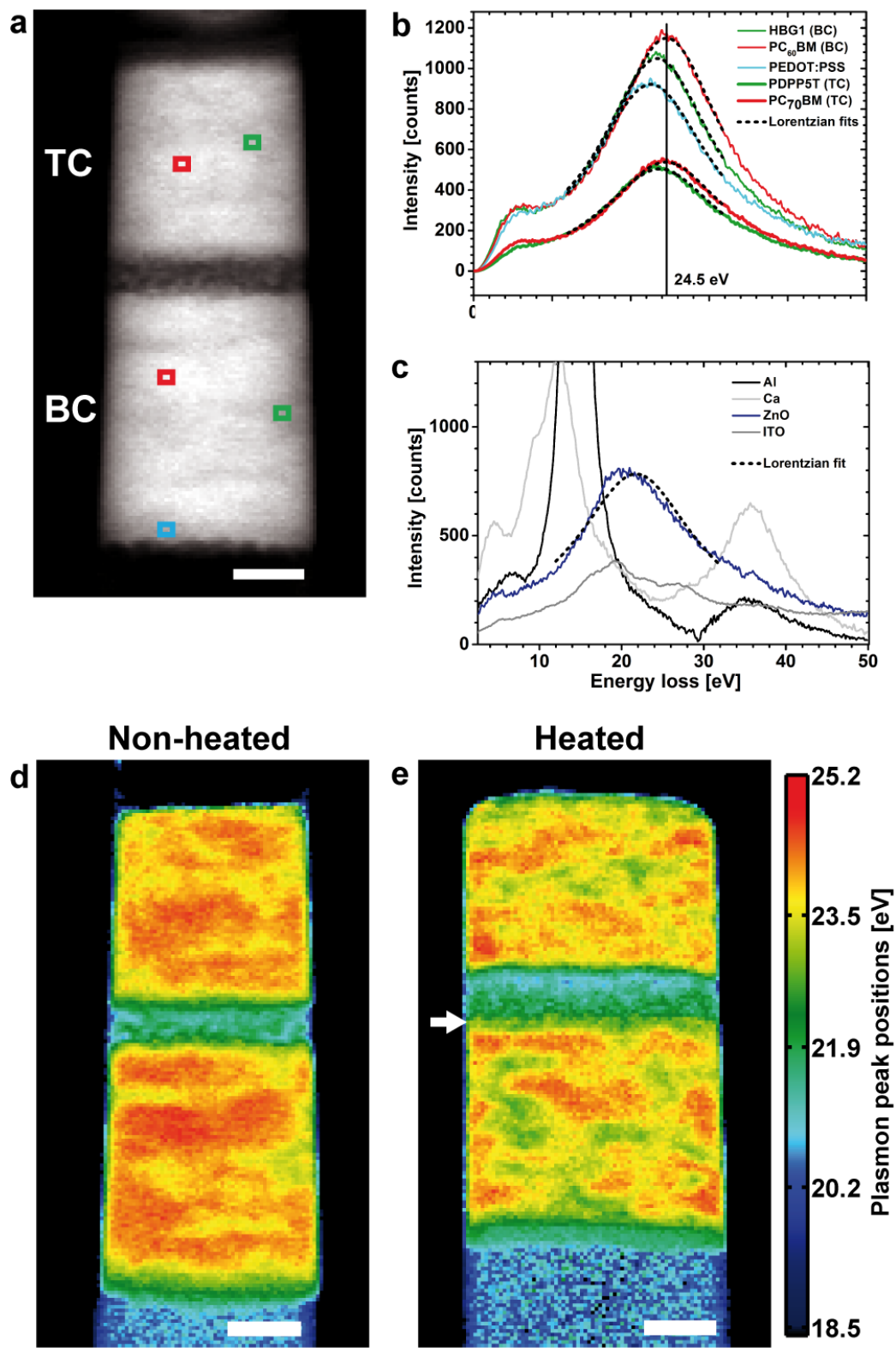

Figure 2: 2D nanomorphology from low-energy-loss spectroscopic imaging of rod-shaped samples. a: Cross-sectional view at energy-loss of $28 \mathrm{eV}$ (window of $\pm 2 \mathrm{eV}$ ). A separation of different material compositions in photoactive layers becomes apparent (TC - top cell, BC - bottom cell). b: Low-energy-loss spectra for HBG1-rich and PC ${ }_{60} B M-r i c h$, and PEDOT:PSS areas of the bottom cell as well as PDPP5T-rich and PC $_{70} \mathrm{BM}$-rich areas of the top cell as indicated in a. Spectra from the top cell were divided by two to facilitate comparison. Specific differences between different materials are observed, with very similar signals when comparing polymers or fullerenes from the two cells (black line shows plasmon peak position of fullerene-rich areas from both cells). Differences between fullerene-rich spectra and PEDOT:PSS are even more pronounced. The latter forms a pure layer on top of the ITO electrode. This shows that for the photoactive layers there are contributions from several material domains in the projected data. Volume plasmon peaks were fitted using Lorentzian profiles, which accurately describe these peaks ${ }^{25}$. c: Low-energy-loss spectra for the different inorganic materials. A Lorentzian fit is also shown for ZnO. However, due to various contributions of different signals from the projection an exact fit as for the organic layers is not possible. d,e: Maps of plasmon peak positions fitted using a Lorentzian function for each spectrum from low-energy-loss spectroscopic imaging data sets. Red correlates with fullerene-rich areas and green with polymer-rich areas. Pure PEDOT:PSS correlates with dark green to light blue colours. The arrow in e marks the upper surface region of the bottom cell in the vicinity of the $\mathrm{ZnO}$ part of the recombination layer. $\mathrm{PC}_{60} \mathrm{BM}$, which acts as electron conductor towards $\mathrm{ZnO}$, appears to be reduced in this region, which is not observed for the non-heated case. This would explain reduction in efficiency upon heating. Scale bars in a,d-e represent $50 \mathrm{~nm}$. 
The 2D maps in Figure 2 only provide information from the projection through the 3D object. A solution to this problem is to use ST of plasmon spectra to generate 3D maps. To obtain an ST tilt series of the heated specimen, a low-energy-loss spectral image set was recorded for each $4^{\circ}$ over the tilt range of $\pm 90^{\circ}$ with an energy channel size of $100 \mathrm{meV}$ and a step size of $2.5 \mathrm{~nm}$. Supplemental Figure S2 illustrates the principles of ST experiments for a rod-shaped specimen. After individually reconstructing each energy channel, a 4D reconstruction is obtained where each voxel in 3D represents a reconstructed spectrum. This reconstruction was performed based on 50 iterations of the GPU implementation for the simultaneous iterative reconstruction technique (SIRT) using the ASTRA Tomography Toolbox ${ }^{29}$. Volume plasmon excitations of carbon-based materials have been proven to fulfil the projection requirement ${ }^{30}$ but for the investigated materials, differences are still poorly pronounced for small compositional variations $^{20}$. Therefore-as in the case of $2 \mathrm{D}$ imaging-we fitted Lorentzians to the generated 4D data set. Figure 3a shows a rendering of the 3D plasmon peak mapping in the photoactive layers of the heated tandem cell device together with the $\mathrm{ZnO}$ layer. Bright and dark areas correspond to the fullerene-rich and polymer-rich domains, respectively. Figure $3 \mathrm{~b}$ presents three orthogonal slices (orthoslices) through the reconstruction of the rod. False-colour maps are again used to indicate the varying domain compositions. Averaged low-energy-loss spectra corresponding to different domains (colours) are depicted in Figure 3c, where the green, red and light blue spectra represent polymerenriched, fullerene-enriched and PEDOT:PSS areas, respectively. The spectra resulting from the reconstruction are in very good agreement to the spectra obtained from a 2D mapping. This implies that spectral features that depend on the material composition at specific voxels are reliably reconstructed. Figure $3 d$-f shows reconstruction results for the non-heated rod-shaped specimen, where at first glance no significant differences to the heated sample are observed that could be related to a decrease in cell efficiency. 

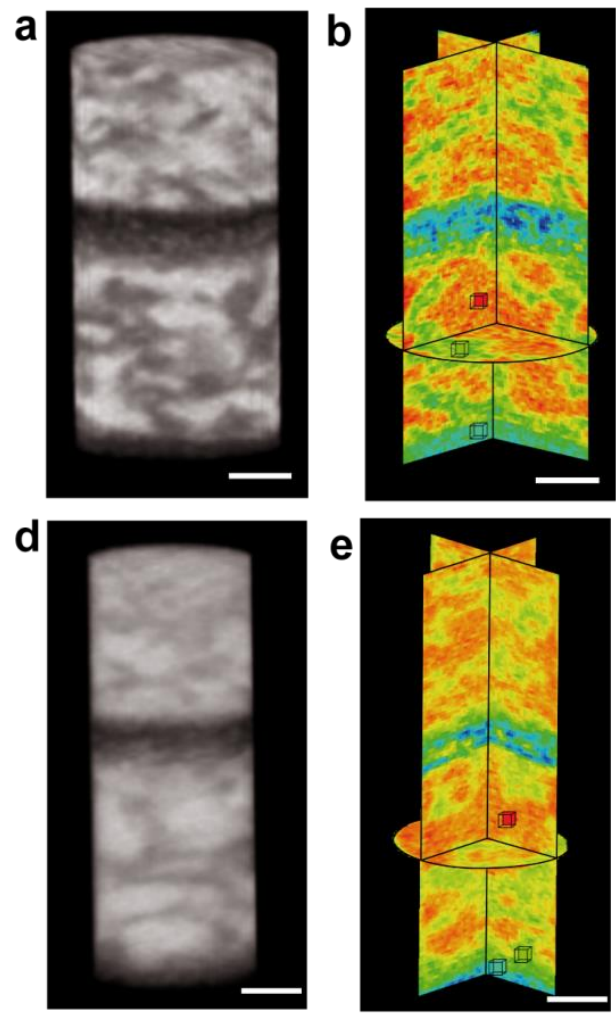
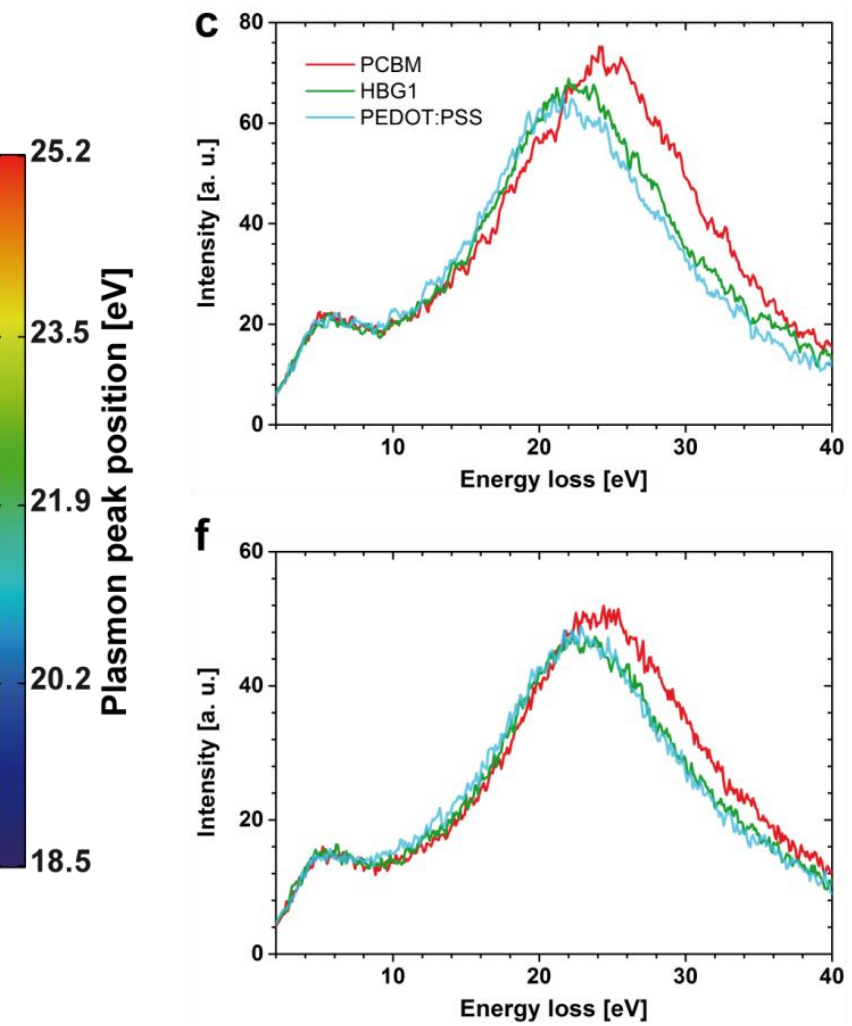

Figure 3: Reconstruction of nanoscale sample morphology by 3D plasmon peak mapping. a: Volume rendering of the rod from the heated tandem cell. The grey colour map encodes varying plasmon peak energies determined by fitting of the reconstructed 4D spectroscopic data set. Bright regions in the photoactive layers correspond to fullerene-rich domains and dark regions to polymer-rich domains. $\mathbf{b}$ : Orthoslices in $X Y, X Z$, and $Y Z$ directions through the reconstruction. False-colours indicate different domain contributions as shown in Figure 2 for the $2 \mathrm{D}$ analysis, with red for fullerene-rich, green for polymer-rich, and blue for PEDOT:PSS or ZnO areas, respectively. c: Average low-energy-loss spectra from three regions (HBG1-enriched, PC $_{60} \mathrm{BM}$ enriched, and PEDOT:PSS) as indicated by the cubes in $\mathbf{b}$. $\mathbf{d}$-f: Identical analysis and illustration for a reconstruction of the nonheated sample. Scale bars in $\mathbf{a}-\mathbf{b}$ and $\mathbf{d}$-e represent $50 \mathrm{~nm}$.

However, the 3D maps presented in Figure 3 enable more detailed investigations of nanostructures of the two samples at a resolution of below $7 \mathrm{~nm}$ (see Methods section for determination of 3D resolution). To correlate the structures with charge transport properties close to the $\mathrm{ZnO}$ (recombination) layer, we segmented $\mathrm{ZnO}$ voxels and fullerene-enriched voxels using the histogram of plasmon peak energies. This allowed computing smallest distances between $\mathrm{ZnO}$ voxels and areas of high fullerene content. Figure $4 \mathrm{a}$ and $4 \mathrm{~b}$ shows false-colour maps of minimal distances using identical segmentation parameters for both samples. The relative frequencies of occurring distances are plotted in Figure 4c. According to these distributions, values for the average and minimal distances of the fullerene-enriched domains are larger for the heated sample compared to the non-heated sample. Such 
conclusions could not be made from 2D projection images but are only enabled by the 3D reconstruction free of missing wedge artefacts. For the heated sample, fullerene-rich voxels are only found at a distance starting at around $4 \mathrm{~nm}$. At distances between 5-10 nm, the fraction of high fullerene content is still very low compared to the non-heated case. Assuming that the sampled volume of the investigated heated rod is representative for the original layer, the observation implies that electrons have to travel through more than $10 \mathrm{~nm}$ of blend material of high HBG1 content to reach the recombination layer. This explains the reduction in performance upon heating in the particular solar cell stack, which can be attributed to space charge accumulations.

An analysis of the recombination layer is provided in Supplemental Figure S3 with segmentations of the PEDOT:PSS and ZnO materials. It can be seen that the PEDOT:PSS follows the ZnO pore geometry, which is beneficial for recombination of holes transferred from the top cell through PEDOT:PSS and electrons from the bottom cell through $\mathrm{ZnO}$. Another insight from the detailed information obtained from 3D spectral imaging is presented in Figure S4. A relatively large plasmon peak energy of $22.5 \mathrm{eV}$ was determined for the PEDOT:PSS spectrum from the projection data in Figure 2. Figure S4 shows three consecutive $X Y$ slices from the reconstruction. In an outer shell of ca. $10 \mathrm{~nm}$, higher plasmon peak energies are observed, which are uncommon for pure polymers. However, the central part of these slices reveal an average plasmon peak energy of ca. $21.8 \mathrm{eV}$, which is in the expected range. This observation implies that FIB preparation induced increased damage at the outer interface of the rod specimens, which could not be examined before with 2D spectral imaging.

Furthermore, Figures S5-7 show analyses of the image features for successive XY-slices. It is observed that slightly smaller domain sizes are located in the central parts of the photoactive layers as compared to interfacial areas. Domains in the centres of the layers are still larger than $10 \mathrm{~nm}$ in size, which are not considered to be too small for efficient charge separation ${ }^{31}$. However, size measurements as 
demonstrated in the supporting information will help to decipher thermodynamic behaviour of other material systems affected, for example, by spinodal decompositions ${ }^{32}$.

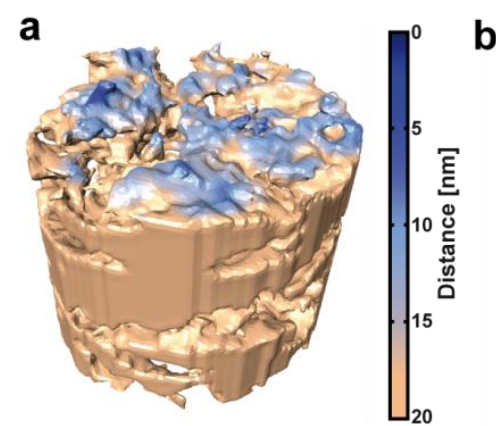

b

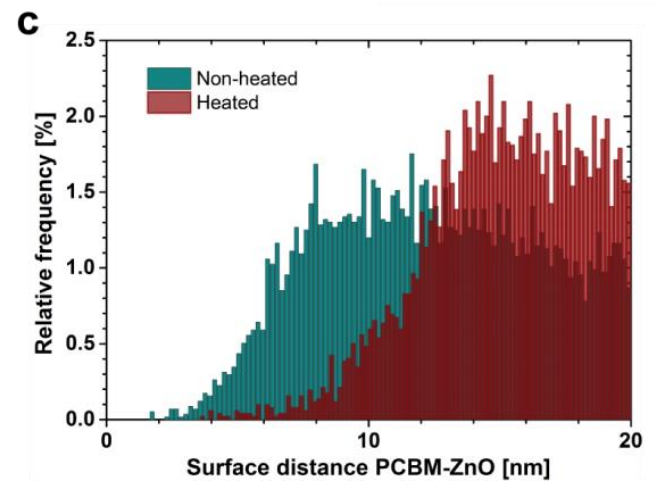

Figure 4: Quantification of 3D distances from $\mathrm{ZnO}$ towards the nearest voxel of $\mathrm{PC}_{60} \mathrm{BM}$-enrichment within the bottom cell. a: Volume rendering of distances between the segmented $\mathrm{ZnO}$ surface and a nearest fullerene-enriched voxel for the heated sample. The colour coding shows that only a very small fraction is separated by less than $10 \mathrm{~nm}$. $\mathbf{b}$ : The same rendering for the non-heated sample reveals larger areas with a distance and that minimum distances are around $5 \mathrm{~nm}$. This allows facilitated transport of electrons towards the recombination layer, particularly at the areas indicated by the arrows. c: Histograms of distances shown in $\mathbf{a}$, and $\mathbf{b}$ up to $20 \mathrm{~nm}$. A comparison between the heated and non-heated samples clearly shows that more $\mathrm{PC}_{60} \mathrm{BM}$ is found near the recombination layer for transfer of electrons generated in the bottom cell.

In a recent study 3D segmentations of free-standing layers into different material domains were shown using HAADF-STEM, on the basis of endohedral fullerenes with an incorporated lutetium atom to enhance contrast ${ }^{33,34}$. Applying ST allows us to obtaining 3D compositional maps of native blends within the particular device structure. Such maps provide fundamental information on the blends with respect to their thermodynamic and optoelectronic properties. For this reason we determined voxels in the reconstruction of each cell with plasmon peaks related to pure polymer and pure fullerene. The spectral information for these voxels is used to generate reference spectra that serve as input to determine the compositional ratios by multiple least-squares fitting ${ }^{35}$. These reference spectra are shown in green and red in Figure 5a for the two cells of the heated sample. Subsequently, reference spectra are fitted to all 
spectra of the $4 \mathrm{D}$ data set to calculate the fractional composition in volume per cent. Sample fits to single spectra with corresponding root-mean-square errors are provided in Supplemental Figure S8. Figure $5 b$ shows three orthogonal slices through the volume of computed polymer fractions. Here, the outer shell of $10 \mathrm{~nm}$ damaged material as determined in Figure $\mathrm{S} 4$ was removed for analysis. By plotting histograms of polymer compositions as illustrated in Figure 5c, two preferential compositions are found with peaks at $64 \%$ and at $20 \%$ polymer content (equal to $80 \% \mathrm{PC}_{60} \mathrm{BM}$-enrichment) for the bottom cell. This result reveals that polymer enrichment occurs at lower concentrations of HBG1 compared to the $\mathrm{PC}_{60} \mathrm{BM}$-enriched domains. It confirms that for a sufficient $\mathrm{PC}_{60} \mathrm{BM}$ concentration, these fullerenes tend to form pure or strongly enriched domains ${ }^{36}$. Polymer domains were segmented applying thresholds of $50 \%$ and $64 \%$ of polymer fraction followed by determination of connected domains. Resulting colourcoded 3D renderings of these domains, presented in Figure $5 \mathrm{~d}$ for both cells, indicate that when applying a threshold of $64 \%$, the top cell shows a stronger fragmentation in comparison to the bottom cell. Whereas the bottom cell yields only one domain that is almost completely connected throughout the whole layer, the upper cell shows a fragmentation into 30 small polymer-rich domains. This observation can be explained by the less pronounced separation of preferential polymer compositions for PDPP5T and $\mathrm{PC}_{70} \mathrm{BM}$ in the top cell. There is indeed no apparent peak at high polymer concentrations. This finding reveals a crucial difference between the two applied material systems within the device. In the upper cell a PCBM fullerene cage of 70 carbon atoms is used while the bottom cell contains fullerene cages of 60 carbon atoms. The quantitative analysis unambiguously provides the measures to which extent differences in demixing at the nanoscale persist in the upper cell. This can be due to the larger fullerene cages that inhibit diffusion in the polymer matrix. Although the connectivity for charge transport is reduced, the diffusion behaviour renders this material system more thermally stable than $\mathrm{HBG1}: \mathrm{PC}_{60} \mathrm{BM}$ and explains why no significant changes upon heating were found here. 


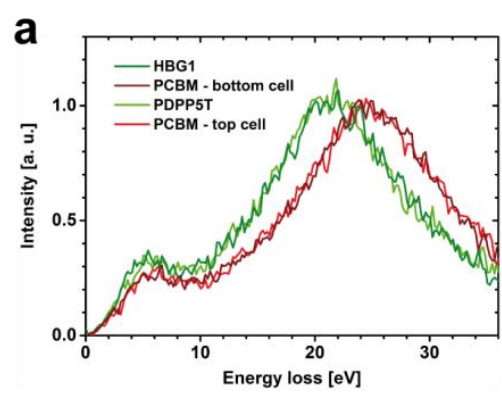

b

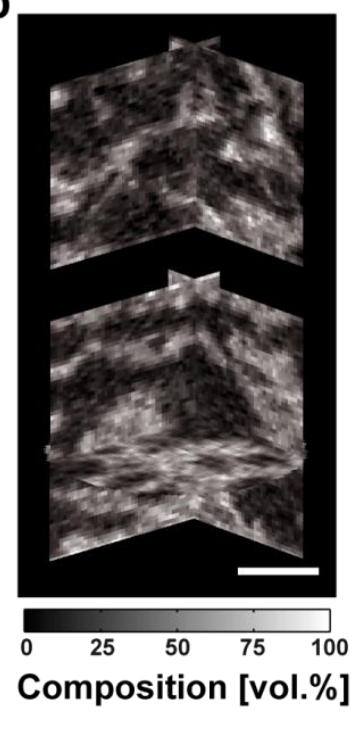

d Connected polymer segments

Composition $>=\mathbf{5 0} \%$

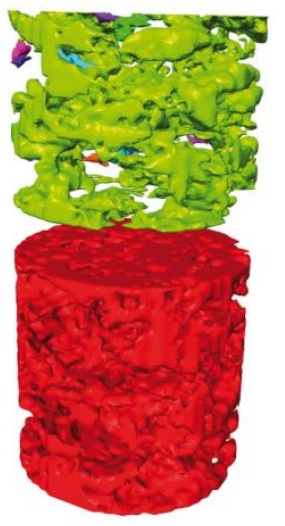

Composition $>=64 \%$

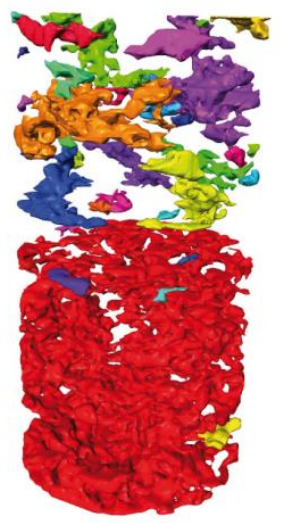

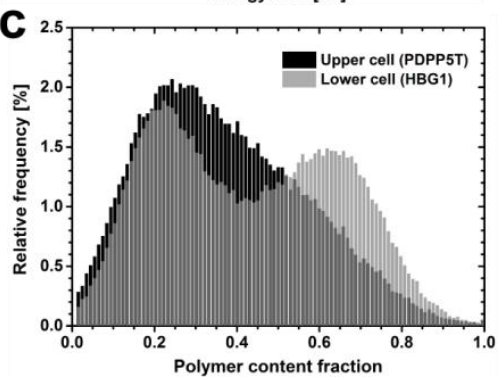

Figure 5: 3D quantification of the bottom and top cell compositional distributions for the heated sample. a: Average reference spectra from the top and bottom cell for both polymers and fullerenes. These are used for a multiple least-squares optimization to determine the linear contributions of polymer and fullerene spectra for each spectral voxel. The fitting results in a quantified 3D representation of the photoactive layers with the contributions of both materials. $\mathbf{b}$ : Orthoslices in $X Y, X Z$, and YZ directions showing the top and bottom cells represented by a mapping of polymer fraction in per-cent for each voxel. c: Histograms of compositions for the top and bottom cells. It can be seen for the bottom cell that $\mathrm{PC}_{60} \mathrm{BM}$-enriched areas show a high fullerene content peaking at $80 \% \mathrm{PC}_{60} \mathrm{BM}$ fraction whereas $\mathrm{HBG1}$-enriched areas show a peak at around $65 \%$ HBG1 fraction. In the top cell less demixing is observed. $\mathbf{d}$ : 3D rendering of connected polymer-rich domains from segmentation using thresholds of $50 \%$ (left) and $64 \%$ (right) polymer content. Both top (yellow segment) and bottom cells (red segment) mainly contain one large component with $50 \%$ polymer content or higher. Considering only voxels of $64 \%$ and higher, the top cell shows stronger fragmentation into smaller, non-connected polymer domains. The bottom cell still contains mainly one polymer-rich segment. Scale bar in b represents $50 \mathrm{~nm}$.

Spatially resolved spectral imaging in TEM has been a valuable tool to reveal insights into organic blend morphologies, particularly when results are complemented with other techniques such as X-ray imaging $^{37}$ and atomic force microscopy ${ }^{38}$. However, for a 3D visualization of chemical compositions in organic blends at high resolution, several limitations have to be overcome: (i) weak specific material contrast in case of poor phase separation, (ii) electron irradiation damage and deformation, and (iii) the requirement for visualizing complete devices. We applied a technique that enables solving all of these requirements at sub-10 $\mathrm{nm}$ resolution-and at the same time provides material specific information from plasmon spectra for compositional mapping. It must be noted that the rod-shape, with photoactive layers fixed between inorganic layers, prevents sample deformation and thus enables high effective resolution. Contrast is created using fully reconstructed low-energy-loss spectra from 4D data sets without missing-wedge artefacts. During data collection, damage of organic materials can never be 
completely prevented. However, in Supplemental Figure S9 2D plasmon peak maps of the heated sample at the start of the tilt series and after $180^{\circ}$ tilt are presented, showing only minor changes in peak energies. Furthermore, it is known that even the damage products of polymers and fullerene derivatives show varying plasmon excitations ${ }^{39}$. When starting ST acquisitions of FIB prepared specimens, we can assume that changes towards these products have already commenced due to FIB induced damage and electron beam damage arising at the early stages of the experiment. This is even an advantage for reliable signal comparison since notable changes occur during initial damage of electronic structures. It is therefore crucial to only include data in the tomographic reconstruction and quantification using spectra that do not relate to the initial state of the materials.

The ST approach we introduce in this article yields compositional 3D maps from native organic blends in realistic devices. Since full spectral information is available in 3D, the contribution of both materials for each voxel can be estimated. Reference spectra were generated from the data. Their plasmon peak energies are around $22 \mathrm{eV}$ and $25 \mathrm{eV}$ for polymer and fullerene references, respectively. These values are very similar to expected values after initial damage ${ }^{20}$ so that it can be assumed that they represent pure areas. A multiple least-squares fitting results in 3D compositional maps enabling determination of preferential enrichments at nanoscale resolution. Compositional mapping in both cells of the heated sample illustrates why fullerene content is reduced near the $\mathrm{ZnO}$ layer. The upper cell containing larger $\mathrm{C}_{70}$ fullerene cages still exhibits a stronger degree of mixing after thermal heating compared to the bottom cell with smaller $\mathrm{C}_{60}$ fullerene cages. It implies that for $\mathrm{HBG1}: \mathrm{PC}_{60} \mathrm{BM}$, processing conditions have to be identified that render this material system thermally more stable. Such a quantitative mapping applied to other high-performing material systems will drastically improve the understanding of stateof-the art blends for organic electronics. 


\section{Methods}

\section{Device fabrication}

All materials used except PEDOT:PSS Al4083 (purchased from Clevios), $\mathrm{PC}_{60} \mathrm{BM}$ and $\mathrm{PC}_{70} \mathrm{BM}$ (purchased from Solenne) were received from partners within the Sunflower project. All layers except the electrodes were deposited by blade-coating in air. PEDOT:PSS AI4083 was filtered and deposited on an ITO-coated (150 nm, from Geomatec) glass substrate with a thickness of approximately $20 \mathrm{~nm}$ and annealed for $15 \mathrm{~min}$ at $160^{\circ} \mathrm{C}$.

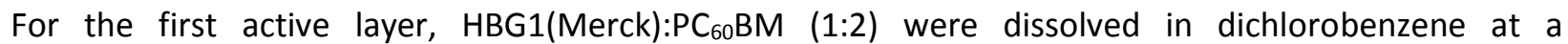
concentration of $30 \mathrm{mg} / \mathrm{ml}$ and deposited on the PEDOT coated substrate with a thickness of ca. $155 \mathrm{~nm}$ and baked for $2 \mathrm{~min}$ at $70{ }^{\circ} \mathrm{C}$. Subsequently, a ZnO nanoparticle solution (GenesInk) was deposited on top of the active layer to obtain a thin $20 \mathrm{~nm}$ layer. A neutral PEDOT:PSS (NT5, AGFA) was deposited on the ZnO layer with a thickness of 10-15 nm. Both ZnO and NT5 were baked for $30 \mathrm{~s}$ at $75{ }^{\circ} \mathrm{C}$. For the top active layer, PDPP5T(BASF):PC $C_{70} B M$ (1:2) were dissolved in a 4:1 mixture of chloroform and dichlorobenzene at a concentration of $30 \mathrm{mg} / \mathrm{ml}$ and deposited to obtain a ca. $110 \mathrm{~nm}$ thick layer. Finally, the coatings were transferred into a glovebox and completed with a $30 \mathrm{~nm} \mathrm{Ca} / 100 \mathrm{~nm}$ Aluminium top electrode deposited by thermal evaporation. The solar cell parameters where measured using a calibrated solar simulator with illumination through a $1 \mathrm{~cm}^{2}$ shadow mask. The two cells (internal references: PP087D1 and PP084D2) used for morphological studies had a power conversion efficiency of $6.1 \%$ and $7.2 \%$, an open-circuit voltage of $1.20 \mathrm{~V}$ and $1.31 \mathrm{~V}$, a short-circuit current density of 8.3 $\mathrm{mA} / \mathrm{cm}^{2}$ and $8.6 \mathrm{~mA} / \mathrm{cm}^{2}$, and a fill factor of $61 \%$ and $65 \%$.

\section{STEM Specimen preparation}

Devices were sealed and stored in the glove box until FIB preparation. Before STEM investigations they were opened, and immediately transferred to a DualBeam Helios Nanolab 650 (FEI). Rod-shaped specimens were prepared perpendicular to the layers using down to $8 \mathrm{kV} \mathrm{Ga}$ ions for thinning. Thicknesses of rods in the central part of the tandem cell device stack were approximately 150-180 nm. Damage from ion irradiation is expected, however, no other spectral changes except the ones known from initial electron irradiation damage were found. For spectral tomography the rod was welded onto a needle tip and mounted on a dedicated on-axis rotation tomography holder (Model 2050, Fischione Instruments). For investigation of a heated samples the original device (which had been heated before during fabrication for $2.5 \mathrm{~min}$ ) was heated on a hot plate to $70^{\circ} \mathrm{C}$ for $2 \mathrm{~min}$ in inert atmosphere before FIB preparation. We would like to note that typically heating treatment of organic solar cells is referred to as thermal annealing. However, we showed that only very specific morphological changes occur instead of a general adaptation of the morphology. To focus on this correlation we refer to a non-heated and heated sample. It was shown that storing a solar cell at room temperature under inert atmosphere can lead to an annealing of the structure without any heating ${ }^{40}$.

\section{Electron microscopy}

Microscopic investigations and spectral tilt series were performed using a Titan 60-300 microscope (FEI) equipped with a GIF Enfinium Spectrometer (Gatan) and operated at $200 \mathrm{kV}$. The step size for spectral data sets in scanning transmission mode was set to $2.5 \mathrm{~nm}$ using a dwell time of $0.5 \mathrm{~ms}$. With a beam current of $70 \mathrm{pA}$ the calculated dose for one spectral data set is $350 \mathrm{e} / \AA^{2}$. For ST $4^{\circ}$ tilt angle increments were used for most of the steps, except for the range that contains the overlap between -90 and $90^{\circ}$, 
which cannot be determined more accurately before acquisition. Within this range an increment of $2^{\circ}$ was used resulting in altogether 70 data sets. With this measure the accumulated dose was calculated to be $245 \cdot 10^{2} \mathrm{e} / \AA^{2}$. To reduce memory consumption the dispersion was set to $0.05 \mathrm{eV} / \mathrm{pixel}$ but using a binning on the camera by a factor of 2 . This led to an energy channel size of $0.1 \mathrm{eV} / \mathrm{pixel}$ with 1024 channels/spectrum. For each low-energy-loss spectral data set, a conventional dark-field image was recorded simultaneously to allow spatial alignment of the data for the different tilt angles. The zeroloss peak was included for spectral alignment of the data.

\section{Data processing and visualization}

For both sample series the dark-field images for each tilt angle were aligned by cross-correlation. Overlapping images were identified to extract series of 45 images. The computed transformation parameters were applied to all 1024 channels of the 45 spectroscopic data sets. This is followed by alignment of the spectra of all spatially transformed data sets using a custom script in Matlab (MathWorks). For importing data into Matlab original Gatan dm3 files were converted to 32 bit Tif files by Fiji (ImageJ, NIH). To account for multiple-scattering events depending on the varying thickness across the rods, all spectra were deconvolved by Fourier-log deconvolution with an adapted Matlab script originally provided by R. Egerton (www.tem-eels.ca). 3D reconstruction of low-energy-loss spectra was done with the ASTRA Tomography Toolbox ${ }^{29}$ applying the GPU implementation of the SIRT algorithm using 50 iterations. An energy range of 0.1-50 eV was selected with the raw energy channel size of $0.1 \mathrm{eV} / \mathrm{pixel}$. This results in $4 \mathrm{D}$ volumes with energy-loss spectra for each voxel. For fitting of volume plasmon peak positions the Matlab workspace was further processed with HyperSpy, an opensource Python library for analysis of multidimensional data sets (www.hyperspy.org). This includes Lorentzian fits to determine the plasmon peak energies, and-with the aid of the Imfit packagemultiple least-squares fitting of reference spectra.

Image stacks coding for plasmon peak energies were visualized with Amira (FEI). This includes segmentation of the data, calculation of surface distance maps, and determination of connected domains. For visualization, the volume was cut leaving a rim of approximately $10 \mathrm{~nm}$, which might not be without artefacts in the reconstruction because of influences of surface plasmons near the vacuum edge, and of elevated damage due to ion-beam damage from FIB preparation.

The spatial resolution of the SIRT reconstruction was estimated using the edge between the reconstructed dark-field information of ITO and vacuum in YZ-orthoslices, following a method proposed by Heidari et al. ${ }^{41}$ The purely sampling limited resolution in our case based on the Crowther criterion for the best resolution direction $(\mathrm{Y})$ is the same as the resolution in $2 \mathrm{D}$ projection (i.e. $5 \mathrm{~nm}$ ) and is deteriorated (approx. $10 \mathrm{~nm}$ ) along the other axes (X, Z); however, there is no global estimate for the resolution of a constrained reconstruction technique ${ }^{42}$. To provide a better figure of merit of the real experimental resolution we have used the edge spread function as a directional measure of the point spread function of the iterative reconstruction system. The value for the work at hand was determined to be $7 \mathrm{~nm}$. It should be noted that this is an empirical estimate of the resolution along the principal tomographic axes, particularly for the heterogeneous intensity distribution inside the rod. 


\section{References}

1. Bals, S.; Goris, B.; Liz-Marzán, L. M.; Van Tendeloo, G. Angew. Chem. Int. Ed. 2014, 53, 1060010610.

2. $\quad$ Van Aert, S.; Batenburg, K. J.; Rossell, M. D.; Erni, R.; Van Tendeloo, G. Nature 2011, 470, 374377.

3. Goris, B.; Polavarapu, L.; Bals, S.; Van Tendeloo, G.; Liz-Marzán, L. M. Nano Lett 2014, 14, 32203226.

4. Lewis, E. A.; Slater, T. J. A.; Prestat, E.; Macedo, A.; O'Brien, P.; Camargo, P. H. C.; Haigh, S. J. Nanoscale 2014, 6, 13598-13605.

5. Haberfehlner, G.; Orthacker, A.; Albu, M.; Li, J.; Kothleitner, G. Nanoscale 2014, 6, 14563-14569.

6. Jarausch, K.; Thomas, P.; Leonard, D. N.; Twesten, R.; Booth, C. R. Ultramicroscopy 2009, 109, 326-337.

7. Jin-Phillipp, N. Y.; Koch, C. T.; van Aken, P. A. Ultramicroscopy 2011, 111, 1255-1261.

8. $\quad$ Goris, B.; Turner, S.; Bals, S.; Van Tendeloo, G. ACS Nano 2014, 8, 10878-10884.

9. Nicoletti, O.; de la Peña, F.; Leary, R. K.; Holland, D. J.; Ducati, C.; Midgley, P. A. Nature 2013, $502,80-84$.

10. Yurtsever, A.; Weyland, M.; Muller, D. A. Applied Physics Letters 2006, 89, 151920.

11. Gass, M. H.; Koziol, K. K. K.; Windle, A. H.; Midgley, P. A. Nano Lett 2006, 6, 376-379.

12. Hammond, M. R.; Kline, R. J.; Herzing, A. A.; Richter, L. J.; Germack, D. S.; Ro, H.-W.; Soles, C. L.; Fischer, D. A.; Xu, T.; Yu, L.; Toney, M. F.; DeLongchamp, D. M. ACS Nano 2011, 5, 8248-8257.

13. Herzing, A. A.; Richter, L. J.; Anderson, I. M. J. Phys. Chem. C 2010, 114, 17501-17508.

14. Bulliard, X.; Ihn, S.-G.; Yun, S.; Kim, Y.; Choi, D.; Choi, J.-Y.; Kim, M.; Sim, M.; Park, J.-H.; Choi, W.; Cho, K. Adv. Funct. Mater. 2010, 20, 4381-4387.

15. Karagiannidis, P. G.; Kalfagiannis, N.; Georgiou, D.; Laskarakis, A.; Hastas, N. A.; Pitsalidis, C.; Logothetidis, S. J. Mater. Chem. 2012, 22, 14624.

16. McNeill, C. R. Energy Environ. Sci. 2012, 5, 5653-5667.

17. Biermans, E.; Molina, L.; Batenburg, K. J.; Bals, S.; Van Tendeloo, G. Nano Lett 2010, 10, 50145019.

18. Ke, X.; Bals, S.; Cott, D.; Hantschel, T.; Bender, H.; Van Tendeloo, G. Microsc. Microanal. 2010, $16,210-217$.

19. Jinnai, H.; Spontak, R. J.; Nishi, T. Macromolecules 2010, 43, 1675-1688.

20. Pfannmöller, M.; Kowalsky, W.; Schröder, R. R. Energy Environ. Sci. 2013, 6, 2871-2891.

21. Liu, Y.; Zhao, J.; Li, Z.; Mu, C.; Ma, W.; Hu, H.; Jiang, K.; Lin, H.; Ade, H.; Yan, H. Nat Commun 2014, 5.

22. Bartesaghi, D.; Turbiez, M.; Koster, L. J. A. Org. Electron. 2014, 15, 3191-3202.

23. Li, C.-Z.; Yip, H.-L.; Jen, A. K.-Y. J. Mater. Chem. 2012, 22, 4161-4177.

24. Schindler, W.; Wollgarten, M.; Fostiropoulos, K. Org. Electron. 2012, 13, 1100-1104.

25. Egerton, R. F., Electron Energy-Loss Spectroscopy in the Electron Microscope. 3. ed.; Springer: New York, 2011.

26. Albrecht, S.; Janietz, S.; Schindler, W.; Frisch, J.; Kurpiers, J.; Kniepert, J.; Inal, S.; Pingel, P.; Fostiropoulos, K.; Koch, N.; Neher, D. J. Am. Chem. Soc. 2012, 134, 14932-14944.

27. Buchaca-Domingo, E.; Ferguson, A. J.; Jamieson, F. C.; McCarthy-Ward, T.; Shoaee, S.; Tumbleston, J. R.; Reid, O. G.; Yu, L.; Madec, M.-B.; Pfannmöller, M.; Hermerschmidt, F.; Schröder, R. R.; Watkins, S. E.; Kopidakis, N.; Portale, G.; Amassian, A.; Heeney, M.; Ade, H.; Rumbles, G.; Durrant, J. R.; Stingelin, N. Mater. Horiz. 2014, 1, 270-279.

28. Pfannmöller, M.; Flügge, H.; Benner, G.; Wacker, I.; Kowalsky, W.; Schröder, R. R. Synthetic Met. 2012, 161, 2526-2533. 
29. Palenstijn, W. J.; Batenburg, K. J.; Sijbers, J. J. Struct. Biol. 2011, 176, 250-253.

30. Daniels, H. R.; Brydson, R.; Brown, A.; Rand, B. Ultramicroscopy 2003, 96, 547-558.

31. Lu, L.; Yu, L. Adv. Mater. 2014, 26, 4413-4430.

32. Vaynzof, Y.; Kabra, D.; Zhao, L.; Chua, L. L.; Steiner, U.; Friend, R. H. ACS Nano 2010, 5, 329-336.

33. Roehling, J. D.; Batenburg, K. J.; Swain, F. B.; Moulé, A. J.; Arslan, I. Adv. Funct. Mater. 2012, 23, 2115-2122.

34. Wodo, O.; Roehling, J. D.; Moule, A. J.; Ganapathysubramanian, B. Energy Environ. Sci. 2013, 6, 3060-3070.

35. Hunt, J. A.; Disko, M. M.; Behal, S. K.; Leapman, R. D. Ultramicroscopy 1995, 58, 55-64.

36. Jamieson, F. C.; Domingo, E. B.; McCarthy-Ward, T.; Heeney, M.; Stingelin, N.; Durrant, J. R. Chem. Sci. 2012, 3, 485-492.

37. McNeill, C. R.; Ade, H. J. Mater. Chem. C 2013, 1, 187-201.

38. Bonnell, D. A.; Basov, D. N.; Bode, M.; Diebold, U.; Kalinin, S. V.; Madhavan, V.; Novotny, L.; Salmeron, M.; Schwarz, U. D.; Weiss, P. S. Rev. Mod. Phys. 2012, 84, 1343-1381.

39. Pfannmöller, M.; Flügge, H.; Benner, G.; Wacker, I.; Sommer, C.; Hanselmann, M.; Schmale, S.; Schmidt, H.; Hamprecht, F. A.; Rabe, T.; Kowalsky, W.; Schröder, R. R. Nano Lett 2011, 11, 3099-3107.

40. Guerrero, A.; Pfannmöller, M.; Kovalenko, A.; Ripolles, T. S.; Heidari, H.; Bals, S.; Kaufmann, L.-D.; Bisquert, J.; Garcia-Belmonte, G. Org. Electron. 2015, 16, 227-233.

41. Heidari Mezerji, H.; Van den Broek, W.; Bals, S. Ultramicroscopy 2011, 111, 330-336.

42. Carazo, J.-M., The Fidelity of 3D Reconstructions from Incomplete Data and the Use of Restoration Methods. In Electron Tomography, Frank, J., Ed. Springer US: 1992; pp 117-164.

\section{Supporting Information}

Supporting Information Available: Technical details, analysis of the recombination layer and the PEDOT:PSS layer, domain size distributions, multiple least-squares fitting of energy-loss spectra, and plasmon peak maps for different tilt angles. This material is available free of charge via the Internet at http://pubs.acs.org.

\section{Acknowledgements}

This work was supported by the FP7 European collaborative project SUNFLOWER (FP7-ICT-2011-7contract num. 287594). S.B. acknowledges financial support from European Research Council (ERC Starting Grant \#335078-COLOURATOMS). M.P. gratefully acknowledges the SIM NanoForce program for their financial support. We acknowledge AGFA for providing the neutral PEDOT:PSS and GenesInk for the $\mathrm{ZnO}$ nanoparticles. We would like to thank Stijn Van den broeck for extensive support on FIB sample preparation. M.P. and H.H. thank Daniele Zanaga for the many fruitful discussions.

\section{Author Contributions}

M.P. and H.H. contributed equally to this work. 


\section{Competing Financial Interests}

The authors declare no competing financial interests.

\section{Table of Contents Graphic}
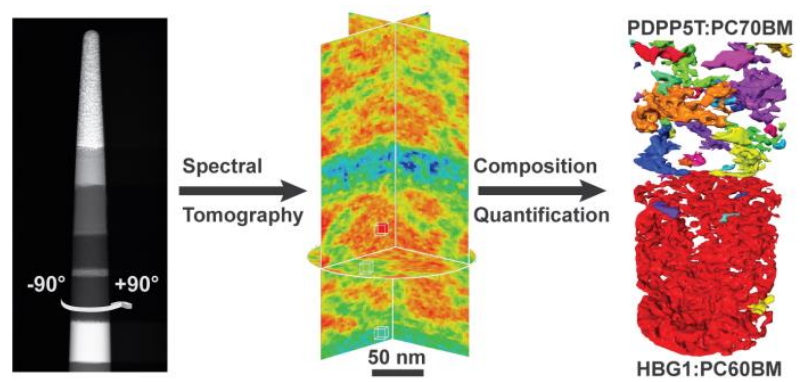